\title{
Carbon dioxide versus room air insufflation during balloon- assisted enteroscopy: A systematic review with meta-analysis
}

\section{(1) $\odot \ominus$}

\section{Authors}

Ashok Shiani ${ }^{1}$, Seth Lipka² ${ }^{2}$ Andrew Lai ${ }^{4}$, Andrea C. Rodriguez ${ }^{1}$, Christian M. Andrade², Ambuj Kumar ${ }^{3}$, Patrick Brady²

Institutions

1 Department of Internal Medicine, University of South Florida, Tampa, Florida, United States

2 Department of Digestive Diseases and Nutrition, University of South Florida, Tampa, Florida, United States

3 Department of Evidence Based Medicine, University of South Florida, Tampa, Florida, United States

4 Morsani College of Medicine Medical School, University of South Florida, Tampa, Florida, United States

submitted 19.5.2016

accepted after revision $\quad 4.10 .2016$

Bibliography

DOI http://dx.doi.org/10.1055/s-0042-118702 |

Endoscopy International Open 2017; 05: E67-E75

(c) Georg Thieme Verlag KG Stuttgart · New York

ISSN 2364-3722

Corresponding author

Ashok Shiani, MD, Morsani College of Medicine, University of South Florida, Department of Internal Medicine, 17 Davis Blvd. Suite 308, Tampa, FL 33606, United States

ashiani@health.usf.edu

\section{ABSTRACT}

Background and study aims Carbon dioxide $\left(\mathrm{CO}_{2}\right)$ insufflation has been suggested to be an ideal alternative to room air insufflation to reduce trapped air within the bowel lumen after balloon assisted enteroscopy (BAE). We performed a systematic review and meta-analysis to assess the safety and efficacy of utilizing $\mathrm{CO}_{2}$ insufflation as compared to room air during BAE.

Patients and methods The primary outcome is mean change in visual analog scale (VAS; $10 \mathrm{~cm}$ ) at 1, 3, and 6 hours to assess pain. Secondary outcomes include insertion depth (anterograde or retrograde), adverse events, total enteroscopy rate, diagnostic yield, mean anesthetic dosage, and $\mathrm{PaCO}_{2}$ at procedure completion. We searched MEDLINE and the Cochrane Central Register of Controlled Trials (CENTRAL) from inception until May 2015. Multiple independent extractions were performed, the process was executed as per the standards of the Cochrane collaboration.

Results Four randomized controlled trials (RCTs) were included in the meta-analysis. VAS at 6 hours favored $\mathrm{CO}_{2}$ over room air (MD $0.13 ; 95 \% \mathrm{Cl} 0.01,0.25 ; \mathrm{p}=0.03)$. Anterograde insertion depth $(\mathrm{cm})$ was improved in the $\mathrm{CO}_{2}$ group (MD, $58.2 ; 95 \% \mathrm{Cl} 17.17$, 99.23; $\mathrm{p}=0.005)$, with an improvement in total enteroscopy rate in the $\mathrm{CO}_{2}$ group (RR 1.91; $95 \% \mathrm{Cl} 1.20,3.06 ; \mathrm{p}=0.007$ ). Mean dose of propofol $(\mathrm{mg})$ favored $\mathrm{CO}_{2}$ compared to air (MD, -70.53 ; $95 \% \mathrm{Cl}-$ $115.07,-25.98 ; P=0.002)$. There were no differences in adverse events in either group.

Conclusions Despite the ability of $\mathrm{CO}_{2}$ to improve insertion depth and decrease amount of anesthesia required, further randomized control trials are needed to determine the agent of choice for insufflation in balloon assisted enteroscopy.

\section{Introduction}

Historically, the small bowel has been difficult to visualize with conventional endoscopic techniques. With the advent of balloon assisted enteroscopy (BAE), both single-balloon enteroscopy (SBE) and double-balloon enteroscopy (DBE) have proven to be effective tools to safely visualize the small bowel [1]. SBE and DBE tend to be longer procedures (95 minutes and 105 minutes, respectively) when compared to other forms of endoscopy, thus they utilize larger volumes of air [1]. Room air insufflation is most commonly used to distend the lumen to achieve ideal visualization of the bowel. However, the use of air is not optimal as large fractions of air remain trapped within the bowel which must pass through the remaining gastrointestinal tract in order to escape [2]. Pain and discomfort commonly occur following these lengthy procedures and are often attributed to the remaining air that continues to distend the bowel $[3,4]$.
Unlike room air, carbon dioxide $\left(\mathrm{CO}_{2}\right)$ is highly diffusible, incombustible, and rapidly absorbed through the bowel wall, qualifying it as an ideal alternative to insufflate the bowel lumen [5]. Dozens of studies have explored the potential advantages and disadvantages of utilizing $\mathrm{CO}_{2}$ in various types of endoscopic procedures. Individual randomized control trials (RCTs) have generated mixed results on outcomes such as procedure times, intubation depth, and abdominal pain and discomfort [6, 7]. Most recently, Wang et al. performed a meta-analysis of the effect of $\mathrm{CO}_{2}$ use on multiple endoscopic procedures but were unable to definitively conclude its potential impact on BAE [5]. Therefore we performed a systematic review and meta-analysis to evaluate the safety and efficacy of $\mathrm{CO}_{2}$ insufflation as compared to room air during BAE. 


\section{Patients and methods}

\section{Selection criteria}

Any randomized controlled trial evaluating the efficacy of $\mathrm{CO}_{2}$ compared to room air in patients undergoing SBE or DBE regardless of publication status (e.g. abstracts, unpublished studies etc.) were eligible for inclusion in the systematic review. Studies that were not a randomized controlled trial, did not have a control, or included specialized treatment groups were excluded. There was no restriction on patient ethnic group or gender.

Five authors (AS, SL, AL, AR, AK) independently extracted data on outcomes from all studies. Data were extracted using a standardized data abstraction form. The same five authors independently reviewed all titles/abstracts and selected studies for inclusion. We included all references that reported results of RCTs of $\mathrm{CO}_{2}$ versus room air in patients undergoing SBE or $\mathrm{DBE}$ in this review.

\section{Types of participants}

We included studies that enrolled participants aged 18 years or older who were scheduled for diagnostic and/or therapeutic balloon assisted enteroscopy.

\section{Outcomes}

Primary outcomes included pain, for which mean change in visual analog scale (VAS; $10 \mathrm{~cm}$ ) at 1, 3, and 6 hours post procedure was used to quantify pain experienced by the patient. Secondary outcomes included insertion depth in cm (anterograde or retrograde), adverse events, total enteroscopy rate, diagnostic yield, mean anesthetic dosage, and $\mathrm{PaCO}_{2}$ at procedure completion.

\section{Search methods}

An electronic search of Cochrane Central Register of Controlled Trials (CENTRAL) and MEDLINE using a combination of MeSH and free text from inception to May 10, 2015 was performed. No language or age limits were used. The following search strategy was utilized: (("single balloon"[MeSH Terms] OR ("single"[All Fields] AND "balloon"[All Fields]) OR "single balloon"[All Fields] OR "single"[All Fields]) AND balloon[All Fields] AND enteroscopy[All Fields]) OR ("double-balloon enteroscopy"[MeSH Terms] OR ("double-balloon"[All Fields] AND "enteroscopy"[All Fields]) OR "double-balloon enteroscopy"[All Fields] OR ("double"[All Fields] AND "balloon"[All Fields] AND "enteroscopy"[All Fields]) OR "double balloon enteroscopy"[All Fields]).

To identify any recently completed studies that have not yet been published in full, we searched conference abstracts from the last 3 meetings (2013-2015) of the American College of Gastroenterology and Digestive Disease Week. We also handsearched references of all identified review articles and included these studies. Finally, in order to identify unpublished or ongoing studies, we searched ClinicalTrials.gov, Roche clinical trial protocol registry (www.roche-trials.com), Novartis clinical trials database (www.novctrd.com), Australian New Zealand
Clinical Trials Registry (ANZCTR), and the metaRegister of Controlled Trials.

\section{Data collection and analysis}

Five authors (AS, AL, SL, AR, AK) reviewed all titles, abstracts, and full-text reports independently. Any disagreements between authors during the study selection were resolved by consensus.

\section{Data extraction and management}

Broadly, we extracted data on author names, location and setting, specific intervention and comparison details, outcomes and participants.

The same 5 authors independently extracted data according to Chapter 7 of the Cochrane Handbook for Systematic Reviews of Interventions using a standardized data extraction form containing the following items (The Cochrane Collaboration. Higgins JPT, Green S. Cochrane, Handbook for Systematic Reviews of Interventions Version 5.1.0. March 2011. In Internet: http:// handbook.cochrane.org; 05/01/2014):

- General information: study title, authors, sources

- Study characteristics: study design, setting, duration of follow-up

- Patient characteristics: number of patients enrolled, number of patients included in the analysis

- Interventions: $\mathrm{CO}_{2}$ vs. air for balloon assisted enteroscopy

- Outcomes: Pain, measured by mean change in visual analog scales (VAS; $10 \mathrm{~cm}$ ) at 1, 3, and 6 hours post-procedure, insertion depths in cm (anterograde or retrograde), adverse events, total enteroscopy rate, diagnostic yield, mean anesthetic dosage, and $\mathrm{PaCO}_{2}$ after procedure.

For studies with multiple publications, we used the publication with longest follow-up for extracting data. Earlier publications were used to extract data on methodology and baseline characteristics. In cases where the method of analysis was not specified by the investigators and only the number of events was reported, we used the number randomized as the denominator, i. e. we recorded results according to intention-to-treat (ITT) analysis.

\section{Assessment of risk of bias}

Five authors (AS, AL, SL, AR, AK) independently assessed the risk of bias in the included studies using The Cochrane Collaboration's tool for assessing the risk of bias as outlined in the Cochrane Handbook for Systematic Reviews of Interventions based on extracted information (The Cochrane Collaboration. Higgins JPT, Green S. Cochrane, Handbook for Systematic Reviews of Interventions Version 5.1.0. March 2011. In Internet: http://handbook.cochrane.org; 05/01/2014). Any disagreements in data extraction were resolved by the senior author (PB). In addition to risk of bias, we evaluated the risk of random error by extracting data on the investigator's pre-determined effect difference, alpha, power, and sample size.

Specifically, for assessment of risk of bias, we graded each component of methodological quality as low, high, or unclear. We evaluated selection bias by assessing the investigators' de- 
scription of method of randomization and allocation concealment. See appendix A for further description of grading.

\section{Unit of analysis issues}

The unit of analysis for this review was individual study. In the case of repeated follow-up (e.g. reporting of results at 3 months and 6 months), we used the longest follow-up from each study. We considered recurring events (e.g. serious adverse events) as a single event that occurred in 1 patient (e.g. 4 instances of pneumonia in 1 patient were counted as 1 patient with pneumonia).

\section{Missing data}

As suggested in the Cochrane Handbook for Systematic Reviews of Interventions in the case of missing outcome data, we made an attempt to contact the principal investigator, corresponding author (or both) of the study (The Cochrane Collaboration. Higgins JPT, Green S. Cochrane, Handbook for Systematic Reviews of Interventions Version 5.1.0. March 2011. In Internet: http:// handbook.cochrane.org; 05/01/2014). If the corresponding author was unable to provide the missing data for an outcome, the study was still included in the systematic review but excluded from the meta-analysis for the outcome with missing data. No imputation of missing individual patient data was undertaken.

\section{Assessment of heterogeneity and reporting biases}

To evaluate heterogeneity between pooled studies, we calculated $X^{2}$ and $I^{2}$ statistics. We considered an $I^{2}>50 \%$ to indicate substantial heterogeneity or a $X^{2}$ test, with the significance level set at $P<0.1$ to indicate statistically significant heterogeneity.

We planned to assess publication bias using a funnel plot if more than 10 studies were included in the review[8]. We evaluated selective reporting of outcomes within studies by comparing outcomes reported with outcomes specified in protocols, when available.

\section{Data synthesis}

We summarized dichotomous data as risk ratio (RR) along with $95 \%$ confidence intervals $(\mathrm{Cl})$ (i.e. clinical and histologic response) and continuous data (i.e. insertion depth, cm) as mean difference (MD) and standard error along with $95 \% \mathrm{Cl}$ using RevMan 5 software (version 5.1.6). We employed a random-effects model using the Der Simonian-Laird approach to pool studies for all analyses [9].

We constructed a Summary of Findings table using the most clinically and patient-relevant outcomes using the Grading of Recommendations Assessment, Development and Evaluation (GRADE) guidelines [10-14]. These outcomes included mean change in visual analog scales (VAS; $10 \mathrm{~cm}$ ) at 1, 3, and 6 hours post-procedure, insertion depths in $\mathrm{cm}$ (anterograde or retrograde), adverse events, total enteroscopy rate, diagnostic yield, mean anesthetic dosage, and $\mathrm{PaCO}_{2}$ after procedure. Additionally, we evaluated and summarized the quality of evidence for each outcome according to Grading of Recommendations Assessment, Development and Evaluation (GRADE) guidelines,

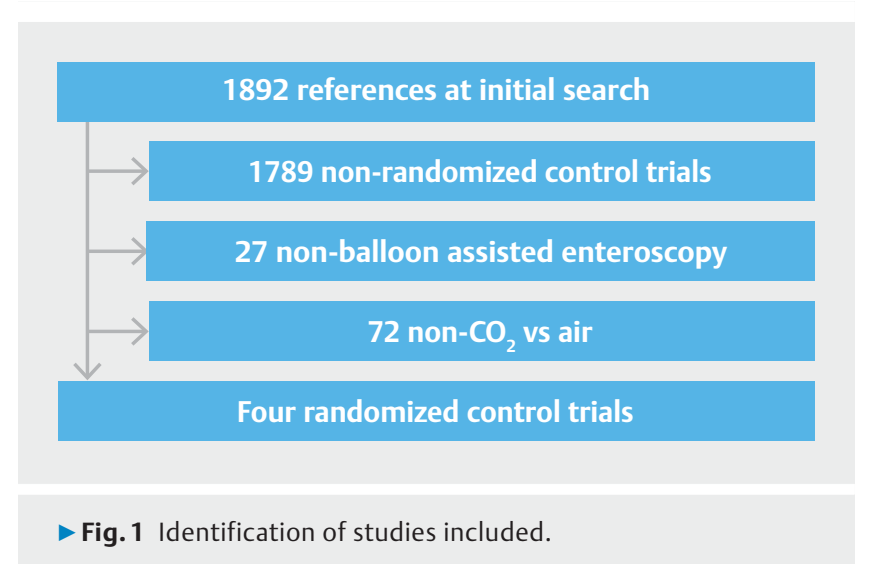

which classify evidence as either very low, low, moderate, or high [10-14]. The systematic review has been performed and reported according to the Preferred Reporting Items for Systematic Reviews and Meta-Analyses (PRISMA) guidelines [15].

\section{Results}

\section{Studies}

The initial search retrieved 1892 references that were screened by title and abstract. Among those 103 were selected for full text review. After the final screening, 4 published studies met

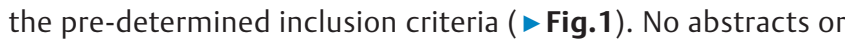
unpublished studies met our inclusion criteria.

\section{Methodologic quality of included studies}

Overall methodologic quality of included studies ranged from moderate to very low.

\section{Effects of interventions}

Our analysis included 4 trials with 461 patients. Overall results for all outcomes and the quality of evidence for the comparison of treatment versus control are summarized in the summary of findings table ( $\triangleright$ Table 1 ). The study design and conclusion of each study are described in ( $\triangleright$ Table 2 ).

\section{Benefits}

Mean VAS score at 1 hour, $\mathrm{CO}_{2}$ vs. air

Data on mean VAS score at 1 hour were available from 4 studies $(n=461)[6,7,16,17]$ The pooled analysis showed no statistical advantage between $\mathrm{CO}_{2}$ and air (MD, $0.10 ; 95 \% \mathrm{Cl}$ to 0.14 , $0.34 ; P=0.43)$; See figure $2 \mathrm{a}$. There was no substantial heterogeneity detected $\left(P=0.78, I^{2}=0 \%\right)$.

\section{Mean VAS score at 3 hours, $\mathrm{CO}_{2}$ vs. air}

Data on mean VAS score at 3 hours were available from 4 studies $(n=461)[6,7,16,17]$. The pooled analysis showed no statistical advantage when comparing $\mathrm{CO}_{2}$ to room air (MD, -0.06 ; $95 \% \mathrm{Cl}-0.41$ to $0.29 ; \mathrm{p}=0.74)$; See $>$ Fig. $\mathbf{2 b}$. There was no substantial heterogeneity detected $\left(P=0.22, \mathrm{I}^{2}=33 \%\right)$. 
- Table 1 Summary of Findings.

\section{Quality assessment}

\begin{tabular}{l|l|l|l|l|}
$\begin{array}{l}\text { No of } \\
\text { studies }\end{array}$ & Design & $\begin{array}{l}\text { Risk } \\
\text { of } \\
\text { bias }\end{array}$ & $\begin{array}{l}\text { Inconsis- } \\
\text { tency }\end{array}$ & $\begin{array}{l}\text { In- } \\
\text { direct- } \\
\text { ness }\end{array}$ \\
\hline
\end{tabular}

Mean VAS at 1 hour (Better indicated by lower values)

\begin{tabular}{|c|c|c|c|c|c|c|c|c|c|c|c|}
\hline 4 & $\begin{array}{l}\text { ran- } \\
\text { dom- } \\
\text { ized } \\
\text { trials }\end{array}$ & $\begin{array}{l}\text { ser- } \\
\text { ious }^{1}\end{array}$ & $\begin{array}{l}\text { no ser- } \\
\text { ious in- } \\
\text { consis- } \\
\text { tency }\end{array}$ & $\begin{array}{l}\text { no ser- } \\
\text { ious in- } \\
\text { direct- } \\
\text { ness }\end{array}$ & $\begin{array}{l}\text { no ser- } \\
\text { ious im- } \\
\text { preci- } \\
\text { sion }\end{array}$ & none & 230 & 231 & - & $\begin{array}{l}\text { MD } 0.1 \\
\text { points } \\
\text { higher } \\
(0.14 \text { to } \\
0.34 \text { ) }\end{array}$ & $\begin{array}{l}\oplus \oplus \oplus \mathrm{O} \\
\text { MODER- } \\
\text { ATE }\end{array}$ \\
\hline
\end{tabular}

Mean VAS at 3 hours (Better indicated by lower values)

\begin{tabular}{|c|c|c|c|c|c|c|c|c|c|c|c|}
\hline 4 & $\begin{array}{l}\text { ran- } \\
\text { dom- } \\
\text { ized } \\
\text { trials }\end{array}$ & $\begin{array}{l}\text { ser- } \\
\text { ious }^{1}\end{array}$ & $\begin{array}{l}\text { no ser- } \\
\text { ious in- } \\
\text { consis- } \\
\text { tency }\end{array}$ & $\begin{array}{l}\text { no ser- } \\
\text { ious in- } \\
\text { direct- } \\
\text { ness }\end{array}$ & $\begin{array}{l}\text { no ser- } \\
\text { ious im- } \\
\text { preci- } \\
\text { sion }\end{array}$ & none & 230 & 231 & - & $\begin{array}{l}\text { MD } 0.06 \\
\text { points } \\
\text { lower } \\
(0.41 \text { to } \\
0.29)\end{array}$ & $\begin{array}{l}\oplus \oplus \oplus O \\
\text { MODER- } \\
\text { ATE }\end{array}$ \\
\hline
\end{tabular}

Mean VAS at 6 hours (Better indicated by lower values)

\begin{tabular}{|c|c|c|c|c|c|c|c|c|c|c|c|}
\hline 4 & $\begin{array}{l}\text { ran- } \\
\text { dom- } \\
\text { ized } \\
\text { trials }\end{array}$ & $\begin{array}{l}\text { ser- } \\
\text { ious }^{1}\end{array}$ & $\begin{array}{l}\text { no ser- } \\
\text { ious in- } \\
\text { consis- } \\
\text { tency }\end{array}$ & $\begin{array}{l}\text { no ser- } \\
\text { ious in- } \\
\text { direct- } \\
\text { ness }\end{array}$ & $\begin{array}{l}\text { no ser- } \\
\text { ious im- } \\
\text { preci- } \\
\text { sion }\end{array}$ & none & 230 & 231 & - & $\begin{array}{l}\text { MD } 0.13 \\
\text { points } \\
\text { higher } \\
(0.01 \text { to } \\
0.25)\end{array}$ & $\begin{array}{l}\oplus \oplus \oplus \mathrm{O} \\
\text { MODER- } \\
\text { ATE }\end{array}$ \\
\hline
\end{tabular}

Mean VAS at 24 hours (Better indicated by lower values)

\begin{tabular}{|c|c|c|c|c|c|c|c|c|c|c|c|}
\hline 3 & $\begin{array}{l}\text { ran- } \\
\text { dom- } \\
\text { ized } \\
\text { trials }\end{array}$ & $\begin{array}{l}\text { ser- } \\
\text { ious } 1\end{array}$ & $\begin{array}{l}\text { no ser- } \\
\text { ious in- } \\
\text { consis- } \\
\text { tency }\end{array}$ & $\begin{array}{l}\text { no ser- } \\
\text { ious in- } \\
\text { direct- } \\
\text { ness }\end{array}$ & $\begin{array}{l}\text { no ser- } \\
\text { ious im- } \\
\text { preci- } \\
\text { sion }\end{array}$ & none & 124 & 123 & - & $\begin{array}{l}\text { MD } 0.11 \\
\text { points } \\
\text { higher } \\
(0.03 \text { to } \\
0.24)\end{array}$ & $\begin{array}{l}\oplus \oplus \oplus \mathrm{O} \\
\text { MODER- } \\
\text { ATE }\end{array}$ \\
\hline
\end{tabular}

Insertion Depth - Anterograde (Better indicated by lower values)

\begin{tabular}{|c|c|c|c|c|c|c|c|c|c|c|c|}
\hline 3 & $\begin{array}{l}\text { ran- } \\
\text { dom- } \\
\text { ized } \\
\text { trials }\end{array}$ & $\begin{array}{l}\text { ser- } \\
\text { ious }^{1}\end{array}$ & serious $^{2}$ & $\begin{array}{l}\text { no ser- } \\
\text { ious in- } \\
\text { direct- } \\
\text { ness }\end{array}$ & serious $^{3}$ & none & 210 & 211 & - & $\begin{array}{l}\text { MD } \\
58.20 \mathrm{~cm} \\
\text { higher } \\
(17.17 \text { to } \\
99.23)\end{array}$ & $\begin{array}{l}\oplus O O O \\
\text { VERY } \\
\text { LOW }\end{array}$ \\
\hline
\end{tabular}

Insertion Depth - Retrograde (Better indicated by lower values)

\begin{tabular}{|c|c|c|c|c|c|c|c|c|c|c|c|}
\hline 3 & $\begin{array}{l}\text { ran- } \\
\text { dom- } \\
\text { ized } \\
\text { trials }\end{array}$ & $\begin{array}{l}\text { ser- } \\
\text { ious }^{1}\end{array}$ & serious $^{4}$ & $\begin{array}{l}\text { no ser- } \\
\text { ious in- } \\
\text { direct- } \\
\text { ness }\end{array}$ & $\begin{array}{l}\text { very } \\
\text { serious }^{3}\end{array}$ & none & 210 & 211 & - & $\begin{array}{l}\text { MD } \\
22.54 \mathrm{~cm} \\
\text { higher } \\
(49.08 \text { to } \\
94.16)\end{array}$ & $\begin{array}{l}\oplus O O O \\
\text { VERY } \\
\text { LOW }\end{array}$ \\
\hline \multicolumn{12}{|c|}{ Insertion Depth Overall (Better indicated by lower values) } \\
\hline 3 & $\begin{array}{l}\text { ran- } \\
\text { dom- } \\
\text { ized } \\
\text { trials }\end{array}$ & $\begin{array}{l}\text { ser- } \\
\text { ious }^{1}\end{array}$ & serious ${ }^{4}$ & $\begin{array}{l}\text { no ser- } \\
\text { ious in- } \\
\text { direct- } \\
\text { ness }\end{array}$ & serious $^{3}$ & none & 120 & 127 & - & $\begin{array}{l}\text { MD } \\
22.96 \mathrm{~cm} \\
\text { higher } \\
\text { (8.82 to } \\
54.74)\end{array}$ & $\begin{array}{l}\oplus O O O \\
\text { VERY } \\
\text { LOW }\end{array}$ \\
\hline \multicolumn{12}{|c|}{ Any adverse Events } \\
\hline 4 & $\begin{array}{l}\text { ran- } \\
\text { dom- } \\
\text { ized } \\
\text { trials }\end{array}$ & $\begin{array}{l}\text { ser- } \\
\text { ious }^{1}\end{array}$ & $\begin{array}{l}\text { no ser- } \\
\text { ious in- } \\
\text { consis- } \\
\text { tency }\end{array}$ & $\begin{array}{l}\text { no ser- } \\
\text { ious in- } \\
\text { direct- } \\
\text { ness }\end{array}$ & $\begin{array}{l}\text { no ser- } \\
\text { ious im- } \\
\text { preci- } \\
\text { sion }\end{array}$ & none & $\begin{array}{l}1 / 230 \\
(0.43 \%)\end{array}$ & $\begin{array}{l}2 / 231 \\
(0.87 \%)\end{array}$ & $\begin{array}{l}\text { RR } 0.63 \\
(0.08 \text { to } \\
4.98)\end{array}$ & $\begin{array}{l}3 \text { fewer } \\
\text { events } \\
\text { per } 1000 \\
\text { (8 to } 34 \text { ) }\end{array}$ & $\begin{array}{l}\oplus \oplus \oplus O \\
\text { MODER- } \\
\text { ATE }\end{array}$ \\
\hline
\end{tabular}


Table 1 (Continuation)

\begin{tabular}{|c|c|c|c|c|c|c|c|c|c|c|c|}
\hline \multicolumn{7}{|c|}{ Quality assessment } & \multicolumn{2}{|c|}{ No of patients } & \multicolumn{2}{|l|}{ Effect } & \multirow[t]{2}{*}{ Quality } \\
\hline $\begin{array}{l}\text { No of } \\
\text { studies }\end{array}$ & Design & $\begin{array}{l}\text { Risk } \\
\text { of } \\
\text { bias }\end{array}$ & $\begin{array}{l}\text { Inconsis- } \\
\text { tency }\end{array}$ & $\begin{array}{l}\text { In- } \\
\text { direct- } \\
\text { ness }\end{array}$ & $\begin{array}{l}\text { Impre- } \\
\text { cision }\end{array}$ & $\begin{array}{l}\text { Other } \\
\text { conside- } \\
\text { rations }\end{array}$ & $\begin{array}{l}\text { Carbon } \\
\text { dioxide } \\
\text { insuf- } \\
\text { flation }\end{array}$ & $\begin{array}{l}\text { Room } \\
\text { air in- } \\
\text { suffla- } \\
\text { tion }\end{array}$ & $\begin{array}{l}\text { Relative } \\
(95 \% \mathrm{Cl})\end{array}$ & Absolute & \\
\hline \multicolumn{12}{|c|}{ Diagnostic Yield } \\
\hline \multirow[t]{2}{*}{2} & \multirow[t]{2}{*}{$\begin{array}{l}\text { ran- } \\
\text { dom- } \\
\text { ized } \\
\text { trials }\end{array}$} & \multirow[t]{2}{*}{$\begin{array}{l}\text { ser- } \\
\text { ious }^{1}\end{array}$} & \multirow[t]{2}{*}{$\begin{array}{l}\text { no ser- } \\
\text { ious in- } \\
\text { consis- } \\
\text { tency }\end{array}$} & \multirow[t]{2}{*}{$\begin{array}{l}\text { no ser- } \\
\text { ious in- } \\
\text { direct- } \\
\text { ness }\end{array}$} & \multirow[t]{2}{*}{$\begin{array}{l}\text { no ser- } \\
\text { ious im- } \\
\text { preci- } \\
\text { sion }\end{array}$} & \multirow[t]{2}{*}{$\begin{array}{l}\text { reporting } \\
\text { bias }^{5}\end{array}$} & $\begin{array}{l}97 / 158 \\
(61.4 \%)\end{array}$ & $\begin{array}{l}91 / 163 \\
(55.8 \%)\end{array}$ & \multirow[t]{2}{*}{$\begin{array}{l}\text { RR } 1.07 \\
(0.8 \text { to } \\
1.43)\end{array}$} & $\begin{array}{l}39 \text { more } \\
\text { per } 1000 \\
(112 \text { few- } \\
\text { er to } 240 \\
\text { more) }\end{array}$ & \multirow[t]{2}{*}{$\begin{array}{l}\oplus \oplus \mathrm{OO} \\
\text { LOW }\end{array}$} \\
\hline & & & & & & & & $60 \%$ & & $\begin{array}{l}42 \text { more } \\
\text { per } 1000 \\
(120 \text { few- } \\
\text { er to } 258 \\
\text { more) }\end{array}$ & \\
\hline \multicolumn{12}{|c|}{ Total Enteroscopy Rate } \\
\hline \multirow[t]{2}{*}{2} & \multirow[t]{2}{*}{$\begin{array}{l}\text { ran- } \\
\text { dom- } \\
\text { ized } \\
\text { trials }\end{array}$} & \multirow[t]{2}{*}{$\begin{array}{l}\text { ser- } \\
\text { ious }^{1}\end{array}$} & \multirow[t]{2}{*}{$\begin{array}{l}\text { no ser- } \\
\text { ious in- } \\
\text { consis- } \\
\text { tency }\end{array}$} & \multirow[t]{2}{*}{$\begin{array}{l}\text { no ser- } \\
\text { ious in- } \\
\text { direct- } \\
\text { ness }\end{array}$} & \multirow[t]{2}{*}{$\begin{array}{l}\text { no ser- } \\
\text { ious im- } \\
\text { preci- } \\
\text { sion }\end{array}$} & \multirow[t]{2}{*}{$\begin{array}{l}\text { reporting } \\
\text { bias }^{5}\end{array}$} & $\begin{array}{l}39 / 158 \\
(24.7 \%)\end{array}$ & $\begin{array}{l}21 / 163 \\
(12.9 \%)\end{array}$ & \multirow[t]{2}{*}{$\begin{array}{l}\text { RR } 1.91 \\
(1.2 \text { to } \\
3.06)\end{array}$} & $\begin{array}{l}117 \text { more } \\
\text { per } 1000 \\
26 \text { more } \\
\text { to } 265 \\
\text { more) }\end{array}$ & \multirow[t]{2}{*}{$\begin{array}{l}\oplus \oplus \mathrm{OO} \\
\text { LOW }\end{array}$} \\
\hline & & & & & & & & $10.6 \%$ & & $\begin{array}{l}96 \text { more } \\
\text { per } 1000 \\
\text { ( } 21 \text { more } \\
\text { to } 218 \\
\text { more) }\end{array}$ & \\
\hline \multicolumn{12}{|c|}{ Sedation - Propofol Dose, Oral DBE (Better indicated by lower values) } \\
\hline 2 & $\begin{array}{l}\text { ran- } \\
\text { dom- } \\
\text { ized } \\
\text { trials }\end{array}$ & $\begin{array}{l}\text { ser- } \\
\text { ious }^{1}\end{array}$ & $\begin{array}{l}\text { no ser- } \\
\text { ious in- } \\
\text { consis- } \\
\text { tency }\end{array}$ & $\begin{array}{l}\text { no ser- } \\
\text { ious in- } \\
\text { direct- } \\
\text { ness }\end{array}$ & $\begin{array}{l}\text { very } \\
\text { serious }\end{array}$ & $\begin{array}{l}\text { reporting } \\
\text { bias }^{5}\end{array}$ & 100 & 107 & - & $\begin{array}{l}\text { MD } \\
70.53 \mathrm{mg} \\
\text { lower } \\
(115.07 \\
\text { to } 25.98)\end{array}$ & $\begin{array}{l}\oplus O O O \\
\text { VERY } \\
\text { LOW }\end{array}$ \\
\hline \multicolumn{12}{|c|}{ Blood Gas - PaCO2 - Anterograde, After DBE (Better indicated by lower values) } \\
\hline 2 & $\begin{array}{l}\text { ran- } \\
\text { dom- } \\
\text { ized } \\
\text { trials }\end{array}$ & $\begin{array}{l}\text { ser- } \\
\text { ious }^{1}\end{array}$ & $\begin{array}{l}\text { no ser- } \\
\text { ious in- } \\
\text { consis- } \\
\text { tency }\end{array}$ & $\begin{array}{l}\text { no ser- } \\
\text { ious in- } \\
\text { direct- } \\
\text { ness }\end{array}$ & $\begin{array}{l}\text { no ser- } \\
\text { ious im- } \\
\text { preci- } \\
\text { sion }\end{array}$ & $\begin{array}{l}\text { reporting } \\
\text { bias }^{5}\end{array}$ & 119 & 120 & - & $\begin{array}{l}\text { MD } 1.2 \\
\text { mmHg } \\
\text { higher } \\
(0.25 \text { to } \\
2.66)\end{array}$ & $\begin{array}{l}\oplus \oplus \mathrm{OO} \\
\text { LOW }\end{array}$ \\
\hline \multicolumn{12}{|c|}{$\begin{array}{l}{ }^{1} \text { Out of } 4 \text { RCTs, } 2 \text { reported method of randomizati } \\
\text { implemented as the endoscopy assistant was res } \\
{ }^{2} \text { Out of } 3 \text { trials, } 2 \text { reported statistically significant } \\
{ }^{3} \text { The results were associated with wide confidence } \\
{ }^{4} \text { The results were conflicting across all } 3 \text { studies } \\
{ }^{5} \text { Out of } 4 \text { trials only } 2 \text { reported this outcome }\end{array}$} \\
\hline
\end{tabular}

Mean VAS score at 6 hours, $\mathrm{CO}_{2}$ vs. air

Data on mean VAS score at 6 hours were available from 4 studies $(n=461)[6,7,16,17]$. The pooled analysis favored $\mathrm{CO}_{2}$ over air (MD 0.13; $95 \% \mathrm{Cl} 0.01$ to $0.25 ; P=0.03$ ); See $>$ Fig. 2c. There was no substantial heterogeneity detected $\left(P=0.53 ; 1^{2}=0 \%\right)$.

\section{Anterograde insertion depth, $\mathrm{CO}_{2}$ vs. air}

Data on mean anterograde insertion depth $(\mathrm{cm})$ were available from 3 studies $(n=261)[6,7,16,17]$. The pooled analysis fa- vored $\mathrm{CO}_{2}$ over air (MD 58.2; $95 \% \mathrm{Cl} 17.17$ to $99.23 \mathrm{P}=0.005$ ); See $>$ Fig.3a. There was substantial heterogeneity detected $(P$ $\left.<0.0001 ;\left.\right|^{2}=89 \%\right)$.

Retrograde insertion depth, $\mathrm{CO}_{2}$ vs. air

Data on mean retrograde insertion depth $(\mathrm{cm})$ were available from 3 studies $(n=421)[6,16,17]$. The pooled analysis showed no statistical difference between $\mathrm{CO}_{2}$ and air (MD 22.54; $95 \%$ 
- Table 2 Summary of studies

\begin{tabular}{|c|c|c|c|c|}
\hline Author & Location & Design & Instrument & Conclusion \\
\hline $\begin{array}{l}\text { Domagk } \\
2007\end{array}$ & Multicenter & $\begin{array}{l}\text { Double Blind } \\
\text { RCT }\end{array}$ & DBE & $\begin{array}{l}\mathrm{CO}_{2} \text { insufflation significantly improved intubation depth, patient discomfort, } \\
\text { diagnostic and therapeutic yield. }\end{array}$ \\
\hline Hirai 2011 & Single Center & $\begin{array}{l}\text { Double Blind } \\
\text { RCT }\end{array}$ & DBE & $\begin{array}{l}\mathrm{CO}_{2} \text { insufflation significantly improved pain, residual gas retention at } 3 \text { hours. } \\
\text { No difference in pre- and post- procedure partial pressure of oxygen or } \mathrm{CO}_{2} \text {. }\end{array}$ \\
\hline Lenz 2013 & Multicenter & $\begin{array}{l}\text { Double Blind } \\
\text { RCT }\end{array}$ & SBE & $\begin{array}{l}\mathrm{CO}_{2} \text { insufflation improved post-procedural pain scores. Insertion depths were } \\
\text { the same between air vs } \mathrm{CO}_{2} \text {, but was significantly greater in the } \mathrm{CO}_{2} \text { group } \\
\text { when looking at patients with previous abdominal surgeries. }\end{array}$ \\
\hline Li 2014 & Single Center & $\begin{array}{l}\text { Double Blind } \\
\text { RCT }\end{array}$ & SBE & $\begin{array}{l}\mathrm{CO}_{2} \text { insufflation improves the intubation depth and total enteroscopy rate in } \\
\mathrm{SBE} \text { with a good safety profile. There was no significant difference between } \\
\mathrm{CO}_{2} \text { and Air in regards to diagnostic yield. }\end{array}$ \\
\hline
\end{tabular}

$\mathrm{Cl}-49.08$ to $94.16 ; \mathrm{p}=0.54)$; See figure $3 \mathrm{~b}$. There was substantial heterogeneity detected $\left(P<0.0001 ; I^{2}=96 \%\right)$.

\section{Overall insertion depth, $\mathrm{CO}_{2}$ vs. air}

Data on mean overall insertion depth $(\mathrm{cm})$ were available from 3 studies $(n=247)[6,7,16,17]$. The pooled analysis showed no significant difference between $\mathrm{CO}_{2}$ and air (MD 22.96; $95 \% \mathrm{Cl}$ 8.82 to $54.74 ; P=0.24)$; See $\boldsymbol{F i g . 3 c}$. There was no substantial heterogeneity detected $\left(P=0.27 ; I^{2}=24 \%\right)$.

\section{Adverse events, $\mathrm{CO}_{2}$ vs. air}

Data on adverse events were available from 4 studies $(n=461)$ $[6,7,16,17]$. However, 2 studies reported zero adverse events for both groups and therefore a summary measure was not derivable $[6,17]$. The pooled analysis with the 2 remaining studies showed no statistical difference between $\mathrm{CO}_{2}$ and air (RR 0.63; $95 \% \mathrm{Cl} 0.08$ to $4.98 ; P=0.66)[7,16]$. There was no substantial heterogeneity detected $\left(P=0.10 ; I^{2}=0 \%\right)$.

\section{Diagnostic yield, $\mathrm{CO}_{2}$ vs. air}

Data on diagnostic yield were available from 2 studies $(n=321)$ $[16,17]$. The pooled analysis showed no statistical difference between $\mathrm{CO}_{2}$ and air (RR 1.07; $95 \% \mathrm{Cl} 0.80$ to $1.43 ; P=0.65$ ). There was no substantial heterogeneity detected $\left(P=0.10 ; I^{2}=\right.$ $63 \%)$.

Total enteroscopy rate, $\mathrm{CO}_{2}$ vs. air

Data on total enteroscopy rates were available from 2 studies $(n=207)[6,17]$. The pooled analysis favored $\mathrm{CO}_{2}$ when compared to room air (RR 1.91; $95 \% \mathrm{Cl} 1.20$ to 3.06; $P=0.007)$. There was no substantial heterogeneity detected $\left(P=0.53 ; I^{2}=\right.$ $0 \%)$.

\section{$\mathrm{CO}_{2}$ vs. air, mean anesthetic dosage $\pm \mathrm{SD}, \mathrm{mg}$}

Data on mean anesthetic dosage $(\mathrm{mg})$, particularly amount of required propofol $(\mathrm{mg})$ during BAE was available from 2 studies $(n=207)[6,17]$. The pooled analysis favored $\mathrm{CO}_{2}$ over air (MD 70.53; $95 \% \mathrm{Cl}-115.07$ to $-25.98 ; P=0.002)$. There was no substantial heterogeneity detected $\left(P=0.29 ; I^{2}=9 \%\right)$.

\section{$\mathrm{CO}_{2}$ vs. air, $\mathrm{PaCO}_{2}$ after procedure}

Data on measured serum $\mathrm{PaCO}_{2}$ after the procedure was available from 2 studies $(n=239)$ [7,16]. The pooled analysis showed no statistical difference between $\mathrm{PaCO}_{2}$ levels between $\mathrm{CO}_{2}$ and air (MD 1.20; $95 \% \mathrm{Cl}-0.25$ to 2.66; $P=0.10$ ). There was no substantial heterogeneity detected $\left(P=0.38 ; I^{2}=0 \%\right)$.

\section{Discussion}

Carbon dioxide has historically been the most common gas used to distend the abdominal cavity to create pneumoperitoneum during laparoscopic abdominal procedures to provide sufficient operating space and adequate visualization [18]. With similar goals during endoscopic procedures, the role of $\mathrm{CO}_{2}$ insufflation in gastrointestinal endoscopy is rapidly evolving.

A previous review by Wang et al. included just 2 RCTs exclusively studying DBE and was unable to determine the advantages of $\mathrm{CO}_{2}$ insufflation [5]. Additional RCTs completed since the previous meta-analysis made updating these results essential. This meta-analysis nearly triples the study population since the previous analysis and is the first to include SBE, the newest form of balloon assisted enteroscopy.

This study found a significant improvement within the $\mathrm{CO}_{2}$ group in anterograde insertion depth, with improved total enteroscopy rates in the subgroup stratification. While retrograde and total insertion depth analysis did not generate significant findings, it is important to note the majority of patients within this review underwent BAE procedures performed through the oral route. This is similar to findings throughout the literature, which not only finds anterograde enteroscopy most popular but also more effective regarding diagnostic and therapeutic yields [19].

We also found a reduction in the average dose of anesthetic, particularly propofol, required during the procedures in patients within the $\mathrm{CO}_{2}$ group, which may clinically suggest a pain reducing effect of $\mathrm{CO}_{2}$ during $\mathrm{BAE}$. Pain assessed using the subjective VAS score was measured at 1, 3, and 6 post- procedure. A reduction in the VAS score of $\mathrm{CO}_{2}$ patients only existed at 6 hours post-procedure. An improvement at a single in- 


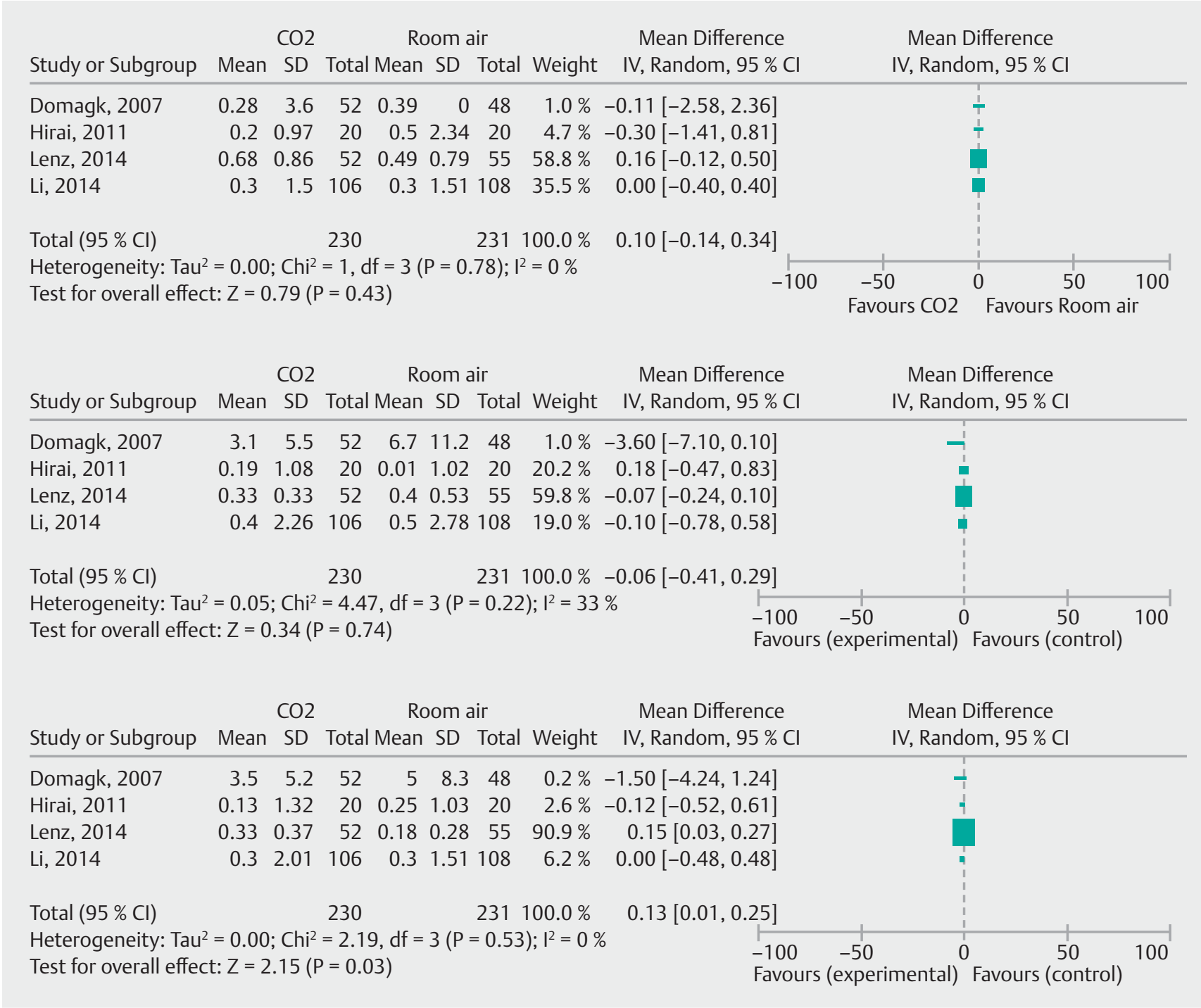

- Fig. 2 a VAS at 1 hour. b VAS at 3 hours. c VAS at 6 hours.

terval of VAS testing is not strong enough evidence to suggest decreased pain with the use of $\mathrm{CO}_{2}$ over air, especially as no difference occurred at either the 1- or 3-hour assessment. No differences emerged regarding the safety of $\mathrm{CO}_{2}$ insufflation compared to air.

A few limitations within this study must be noted. While each study measures several outcomes, not all the numerical data were available to include within this meta-analysis. Secondly several cofounders such as patients with increased likelihood of stenosis, obstruction, or adhesions were not identified in each of the included studies. Depending on the outcome, the quality of evidence ranged from moderate to very low. A metaanalysis in itself has several limitations, which for this study included a limited number of outcomes to measure as studies have to measure the same outcomes in similar formats to be able to compare them using a meta-analysis. The study performed by Lentz et al. due to its size did have a greater weight on the analysis, which is not ideal due to the overall limited number of studies available. Nevertheless, for the main out- comes studied the results were consistent across studies and the quality of evidence was moderate. Lastly using studies that utilized both single balloon and double balloon technique during small bowel enteroscopy is not ideal, however, we feel due to the limited amount of data and number of quality studies in existence currently we gained more power by including both techniques for the inclusion criteria for this meta-analysis.

The inclusion of over 450 patients was a significant strength of this meta-analysis. Four robust RCTs exclusively studying the role of $\mathrm{CO}_{2}$ insufflation during $\mathrm{BAE}$ generated much more statistical power compared to a single study. Lastly, the population was very diverse from multiple centers across the world and did not have significant ethnic disparity. 


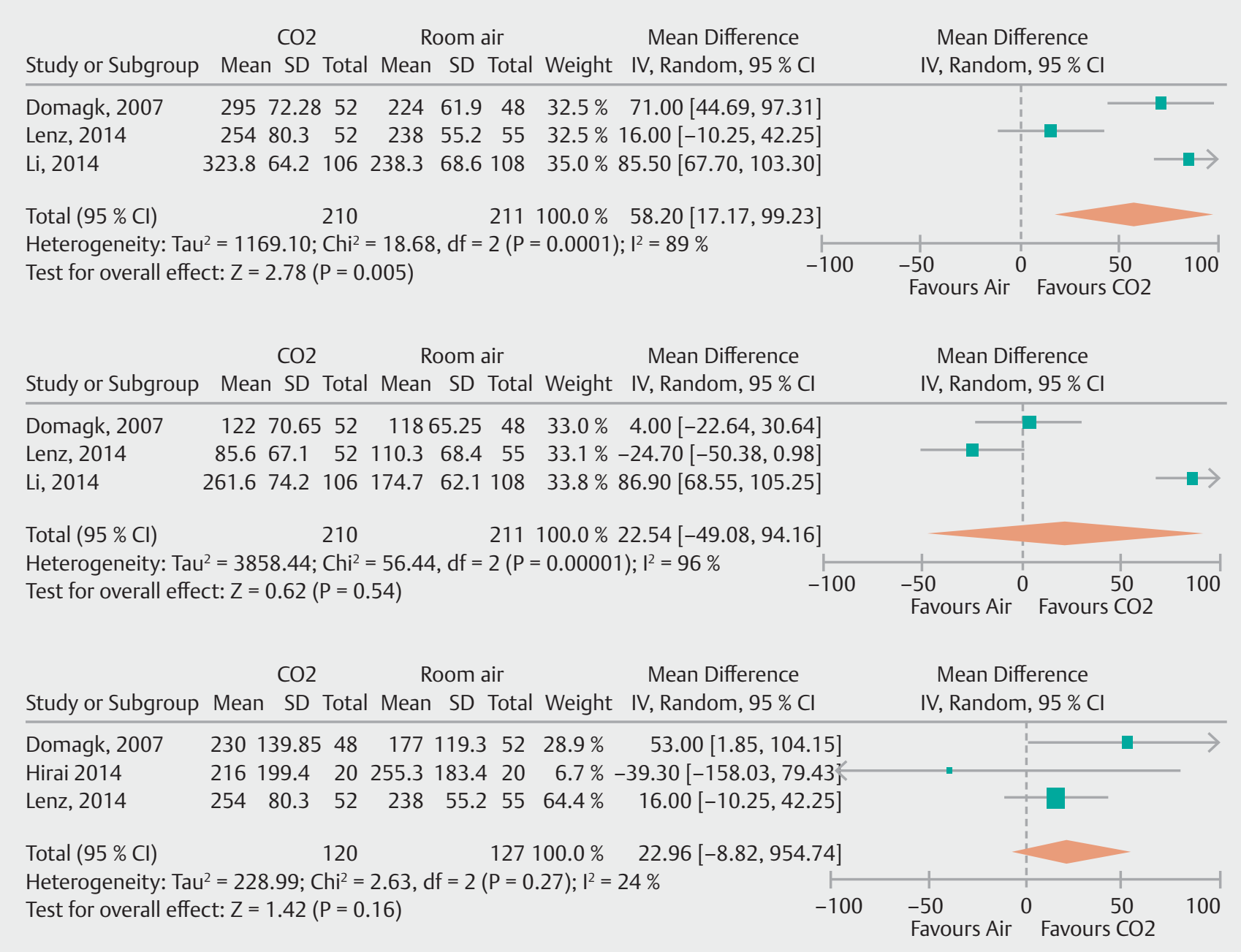

Fig. 3 a anterograde depth. b retrograde insertion depth. c overall insertion depth.

\section{Conclusion}

In conclusion this study determined several potential benefits of utilizing $\mathrm{CO}_{2}$ rather than room air for insufflation during BAE such as the ability to improve insertion depth and decrease the amount of anesthesia required. However, these limited improvements are insufficient to declare $\mathrm{CO}_{2}$ as the agent of choice over room air for insufflation in balloon assisted enteroscopy and further RCTs are needed.

\section{Competing interests}

None

\section{References}

[1] Domagk D, Mensink P, Aktas H et al. Single- vs. double-balloon enteroscopy in small-bowel diagnostics: a randomized multicenter trial. Endoscopy 2011; 43: 472-476
[2] Bretthauer M, Hoff GS, Thiis-Evensen E et al. Air and carbon dioxide volumes insufflated during colonoscopy. Gastrointest Endosc 2003; 58: $203-206$

[3] Dellon ES, Velayudham A, Clarke BW et al. A randomized, controlled, double-blind trial of air vs carbon dioxide insufflation during ERCP. Gastrointestinal endoscopy 2010; 72: 68-77

[4] Church J, Delaney C. Randomized, controlled trial of carbon dioxide insufflation during colonoscopy. Dis Colon Rectum 2003; 46: 322 326

[5] Wang WL, Wu ZH, Sun Q et al. Meta-analysis: the use of carbon dioxide insufflation vs. room air insufflation for gastrointestinal endoscopy. Aliment Pharmacol Ther 2012; 35: 1145-1154

[6] Domagk D, Bretthauer M, Lenz P et al. Carbon dioxide insufflation improves intubation depth in double-balloon enteroscopy: a randomized, controlled, double-blind trial. Endoscopy 2007; 39: 1064 1067

[7] Hirai F, Beppu T, Nishimura T et al. Carbon dioxide insufflation compared with air insufflation in double-balloon enteroscopy: a prospective, randomized, double-blind trial. Gastrointest Endosc 2011; 73: $743-749$

[8] Egger M, Davey Smith G, Schneider M et al. Bias in meta-analysis detected by a simple, graphical test. BMJ (Clinical research ed) 1997; 315: $629-634$ 
[9] DerSimonian R, Laird N. Meta-analysis in clinical trials. Controlled clinical trials 1986; 7: 177-188

[10] Balshem H, Helfand M, Schunemann HJ et al. GRADE guidelines: 3. Rating the quality of evidence. Journal of clinical epidemiology 2011; 64: $401-406$

[11] Guyatt GH, Oxman AD, Kunz R et al. GRADE guidelines 6. Rating the quality of evidence-imprecision. Journal of clinical epidemiology 2011; 64: $1283-1293$

[12] Guyatt GH, Oxman AD, Kunz R et al. GRADE guidelines: 7. Rating the quality of evidence-inconsistency. J Clin Epidemiol 2011; 64: 1294 1302

[13] Guyatt GH, Oxman AD, Montori V et al. GRADE guidelines: 5. Rating the quality of evidence-publication bias. J Clin Epidemiol 2011; 64: $1277-1282$

[14] Guyatt GH, Oxman AD, Vist G et al. GRADE guidelines: 4. Rating the quality of evidence - study limitations (risk of bias). J Clin Epidemiol 2011; 64: 407-415
[15] Moher D, Altman DG, Liberati A et al. PRISMA statement. Epidemiology 2011; 22: 128; author reply 128

[16] Li X, Zhao YJ, Dai J et al. Carbon dioxide insufflation improves the intubation depth and total enteroscopy rate in single-balloon enteroscopy: a randomised, controlled, double-blind trial. Gut 2014; 63: $1560-1565$

[17] Lenz P, Meister T, Manno M et al. CO2 insufflation during single-balloon enteroscopy: a multicenter randomized controlled trial. Endoscopy 2014; 46: $53-58$

[18] Ishizaki Y, Bandai Y, Shimomura K et al. Safe intraabdominal pressure of carbon dioxide pneumoperitoneum during laparoscopic surgery. Surgery 1993; 114: 549- 554

[19] Sanaka MR, Navaneethan U, Kosuru B et al. Antegrade is more effective than retrograde enteroscopy for evaluation and management of suspected small-bowel disease. Clin Gastroenterol Hepatol 2012; 10: $910-916$ 